\title{
Standard Bases for Linear Codes over Prime Fields
}

\author{
Jean Jacques Ferdinand Randriamiarampanahy, Harinaivo Andriatahiny* and \\ Toussaint Joseph Rabeherimanana
}

\begin{abstract}
It is known that a linear code can be represented by a binomial ideal. In this paper, we give standard bases for the ideals in a localization of the multivariate polynomial ring in the case of the linear codes over prime fields.

Keywords: Linear code; semigroup order; Groebner basis; local ring; standard basis.

AMS Subject Classification (2010): Primary: $94 B 05$; Secondary: 12E20; 13P10.

*Corresponding author
\end{abstract}

\section{Introduction}

Coding theory is the mathematical foundation for data transmission through noisy communication channels. It contains two main parts. The first part is to encode the message to reduce its sensitivity to noise during transmission. The second part is to decode the received message by detecting and correcting the errors.

Bruno Buchberger introduced the theory of Groebner bases for polynomial ideals in 1965. The Groebner bases theory can be used to solve some problems concerning the ideals by developing computations in multivariate polynomial rings. In 1964, Hironaka [12] introduced the analogues of Groebner bases called standard bases for ideals in the localization of the polynomial ring at the origin. In [6], standard bases for ideals generated by polynomials in local rings can be determined by using the same method as Groebner bases.

Connection between linear codes and ideals in polynomial rings was presented in [2]. And it was proved that a Groebner basis of the ideal associated to a binary linear code can be used for determining the minimum distance. A generalization to linear codes over prime fields can be found in $[15,16]$. In [15], it has been proved that a linear code can be described by a binomial ideal and a Groebner basis with respect to lexicographic order for the binomial ideal is determined.

The aim of this paper is to present the standard basis of the ideal of a linear code over a prime field in the local ring of rational functions that are regular at a point of the affine variety associated to the ideal. The idea is to generalize the method developed by N. Dück and K. H. Zimmermann in [9].

\section{Preliminaries}

Throughout this paper, $n$ denotes a positive integer, $\mathbb{K}$ a commutative field and $\mathbb{K}[X]:=\mathbb{K}\left[X_{1}, \ldots, X_{n}\right]$ the polynomial ring in $n$ variables over $\mathbb{K}$. We denote by 0 the zero element of $\mathbb{N}^{n}$ where $\mathbb{N}$ is the set of non negative integers.

A monomial in $\mathbb{K}[X]$ is an algebraic expression of the form $X_{1}^{\alpha_{1}} \cdots X_{n}^{\alpha_{n}}$ which is denoted by $X^{\alpha}$ where $\alpha=$ $\left(\alpha_{1}, \ldots, \alpha_{n}\right) \in \mathbb{N}^{n}$. The monomial $X^{\alpha}=X_{1}^{\alpha_{1}} \cdots X_{n}^{\alpha_{n}}$ can be identified with the $\mathrm{n}$-tuple of exponents $\alpha=$ $\left(\alpha_{1}, \ldots, \alpha_{n}\right) \in \mathbb{N}^{n}$ and vice versa, thus there exists a one-to-one correspondence between the monomials in $\mathbb{K}[X]$ and the elements of $\mathbb{N}^{n}$. The degree of the monomial $X^{\alpha}$ with $\alpha=\left(\alpha_{1}, \ldots, \alpha_{n}\right)$ is $|\alpha|:=\alpha_{1}+\alpha_{2}+\cdots+\alpha_{n}$. Any order $>$ we establish on $\mathbb{N}^{n}$ will give us an order on the set of monomials in $\mathbb{K}[X]$ : if $\alpha>\beta$ according to this order, we have $X^{\alpha}>X^{\beta}$. An order $>$ in $\mathbb{K}[X]$ is compatible with multiplication if for all $X^{\alpha}, X^{\beta}$ and $X^{\gamma}$ in $\mathbb{K}[X]$ with

Received : 02-08-2018, Accepted : 06-03-2019 
$X^{\alpha}>X^{\beta}$ then $X^{\alpha} X^{\gamma}>X^{\beta} X^{\gamma}$. Now let $>$ be an order on the set of monomials $X^{u}$ where $u \in \mathbb{N}^{n}$. We say that $>$ is a semigroup order in $\mathbb{K}[X]$ if $>$ is a total order and it is compatible with the multiplication of monomials. A monomial order on $\mathbb{K}[X]$ is a semigroup order such that $1<X_{i}$ for $i=1, \ldots, n$. Usual monomial orders on $\mathbb{K}[X]$ are the lexicographic order, the degree lexicographic order and the degree reverse lexicographic order.

Let $f$ be a non-zero polynomial of $\mathbb{K}[X]$ such that $f=\sum_{i=1}^{k} c_{i} X^{\alpha_{i}}$ where $c_{i} \in \mathbb{K}$ and $\alpha_{i} \in \mathbb{N}^{n}$. Let us fix a monomial order $>$ on $\mathbb{K}[X]$. A term in $\mathbb{K}[X]$ is a scalar times a monomial. The leading term of $f$, denoted by lt $>(f)$, is the largest involved term with respect to $>$. If $\mathrm{lt}_{>}(f)=c_{m} X^{\alpha_{m}}$ where $1 \leq m \leq k$, then $c_{m}$ is called the leading coefficient of $f\left(\mathrm{lc}_{>}(f)\right)$ and $X^{\alpha_{m}}$ is the leading monomial of $f\left(\operatorname{lm}_{>}(f)\right)$. We denote by $\operatorname{deg}(f)$ the maximal degree of all monomials occuring in $f$. The reduction of a polynomial $f$ by a polynomial $g$, denoted by $\operatorname{Red}(f, g)$ is defined by

$$
\operatorname{Red}(f, g):=f-q . g
$$

where $\mathrm{lt}_{>}(f)=q . \mathrm{lt}_{>}(g)$, for some term $q=c X^{\alpha}$. Let $\mathcal{F}=\left(f_{1}, \ldots, f_{s}\right)$ be a s-tuple of polynomials in $\mathbb{K}[X]$. Each polynomial $f \in \mathbb{K}[X]$ can be written in the form :

$$
f=a_{1} f_{1}+a_{2} f_{2}+\cdots+a_{s} f_{s}+r
$$

where $a_{1}, \ldots, a_{s}, r \in \mathbb{K}[X]$ and either $r=0$ or $r$ is a $\mathbb{K}$-linear combination of monomials, none of which is divisible by any of $\mathrm{lt}_{>}\left(f_{1}\right), \ldots, \mathrm{lt}_{>}\left(f_{s}\right)$. Moreover, if $a_{i} f_{i} \neq 0$, then $\mathrm{lt}_{>}(f) \geq \mathrm{lt}_{>}\left(a_{i} f_{i}\right), 1 \leq i \leq s$. The polynomial $r$ is called the remainder of $f$ on division by $\mathcal{F}$. The remainder $r$ is produced by the following algorithm called division algorithm in $\mathbb{K}[X]$ (see [5]).

$$
\begin{aligned}
& \text { Input: } f_{1}, \ldots, f_{s}, f \\
& \text { Output: } r \\
& r:=0 \\
& p:=f \\
& \text { WHILE } p \neq 0 \text { DO } \\
& i:=1 \\
& \text { divisionoccurred: }=\text { false } \\
& \text { WHILE } i \leq s \text { AND divisionoccurred }=\text { false DO } \\
& \text { IF } \mathrm{lt}_{>}\left(f_{i}\right) \text { divides } \mathrm{lt}_{>}(p) \text { THEN } \\
& p:=\operatorname{Red}\left(p, f_{i}\right) \\
& \text { ELSE } \\
& \text { divisionoccurred:= true } \\
& i:=i+1 \\
& \text { IF divisionoccurred }=\text { false THEN } \\
& r:=r+\mathrm{lt}_{>}(p) \\
& p:=p-\mathrm{lt}_{>}(p)
\end{aligned}
$$

The division algorithm terminates after a finite number of steps.

Let $I \subset \mathbb{K}[X]$ be a non-zero ideal and $>$ a monomial order. The ideal generated by the set of $\mathrm{lt}_{>}(f)$ where $f \in I$ is called the leading ideal of $I$, denoted by $l_{>}(I)$, i.e

$$
\mathrm{lt}_{>}(I):=\left\langle\mathrm{lt}_{>}(f) \mid f \in I\right\rangle .
$$

For a finite subset $G=\left\{g_{1}, \ldots, g_{s}\right\}$ of the ideal $I$, we denote by $\mathrm{lt}_{>}(G)$ the ideal generated by the $\mathrm{lt}_{>}\left(g_{i}\right)$, for $i=1, \ldots, s$, i.e

$$
\mathrm{lt}_{>}(G):=\left\langle\mathrm{lt}_{>}\left(g_{1}\right), \ldots, \mathrm{lt}_{>}\left(g_{s}\right)\right\rangle .
$$

A finite subset $G=\left\{g_{1}, \ldots, g_{s}\right\}$ of an ideal $I$ is called a Groebner basis of $I$ if $\mathrm{lt}_{>}(G)=\mathrm{lt}_{>}(I)$. A Groebner basis $G$ for the polynomial ideal $I$ is called a minimal Groebner basis of $I$ if for all $g \in G, \mathrm{lc}_{>}(g)=1$ and $\mathrm{lt}_{>}(g) \notin \mathrm{lt}_{>}(G-\{g\})$. The reduced Groebner basis for the ideal $I$ is a Groebner basis $G$ satisfying :

(1) $\mathrm{lc}_{>}(g)=1$ for all $g \in G$,

(2) no monomial of $g$ lies in $\mathrm{lt}_{>}(G-\{g\})$, for all $g \in G$.

A Groebner basis can be determined by using Buchberger's algorithm. Let $f, g \in \mathbb{K}[X]$ be non-zero polynomials. Fix a monomial order $>$ and let $X^{\gamma}$ be the least common multiple of the leading monomial of $f$ and the leading monomial of $g$. The S-polynomial of $f$ and $g$, denoted by spoly $(f, g)$ is 


$$
\operatorname{spoly}(f, g):=\frac{X^{\gamma}}{\operatorname{lt}_{>}(f)} \cdot f-\frac{X^{\gamma}}{\operatorname{lt}_{>}(g)} \cdot g
$$

If $I$ is a polynomial ideal, then a basis $G=\left\{g_{1}, \ldots, g_{s}\right\}$ for $I$ is a Groebner basis for $I$ if and only if for all pairs $i \neq j$, the remainder on division of $\operatorname{spoly}\left(g_{i}, g_{j}\right)$ by $G$ is zero.

\section{Localization}

Let $\mathbb{K}$ be a commutative field and $p=\left(p_{1}, \ldots, p_{n}\right)$ an n-tuple of $\mathbb{K}^{n} . \mathbb{K}(X):=\mathbb{K}\left(X_{1}, \ldots, X_{n}\right)$ denotes the field of rationnal functions containing $\mathbb{K}[X]$. We define a local ring in $\mathbb{K}(X)$ by the set $\mathcal{O}_{p}:=\left\{\frac{f}{g} \mid f, g \in \mathbb{K}[X], g(p) \neq 0\right\}$. We say also that we localize at the maximal ideal $\left\langle X_{1}-p_{1}, \ldots, X_{n}-p_{n}\right\rangle \subset \mathbb{K}[X]$. Let $m_{p}$ be the ideal generated by $X_{1}-p_{1}, \ldots, X_{n}-p_{n}$ in $\mathcal{O}_{p}$. Then each element in $\mathcal{O}_{p} \backslash m_{p}$ is a unit in $\mathcal{O}_{p}$.

A local order in $\mathbb{K}[X]=\mathbb{K}\left[X_{1}, \ldots, X_{n}\right]$ is a semigroup order such that $1>X_{i}$ for all $1 \leq i \leq n$. For instance, for two n-tuples of $\mathbb{N}^{n} \alpha=\left(\alpha_{1}, \ldots, \alpha_{n}\right)$ and $\beta=\left(\beta_{1}, \ldots, \beta_{n}\right)$, we define the negative degree lexicographic order by $\alpha>\beta$ if $|\alpha|<|\beta|$ or $|\alpha|=|\beta|$ and there exists an integer $i \in\{1, \ldots, n\}$ such that $\alpha_{1}=\beta_{1}, \ldots, \alpha_{i-1}=\beta_{i-1}, \alpha_{i}>\beta_{i}$.

Let $>$ be a local order on the set of monomials of $\mathbb{K}[X]$ and let

$S_{>}:=\left\{1+g \in \mathbb{K}[X] \mid g=0\right.$ or $\left.\operatorname{lt}_{>}(g)<1\right\} . S_{>}$is a multiplicative part of $\mathbb{K}[X]$ and we remark that $S_{>}=$ $\mathbb{K}[X] \backslash\left\langle X_{1}, \ldots, X_{n}\right\rangle$. Define the localization of $\mathbb{K}[X]$ in view of the order $>$ by the ring $([6,13])$

$$
\operatorname{Loc}_{>}(\mathbb{K}[X]):=S_{>}^{-1} \mathbb{K}[X]=\left\{\frac{f}{(1+g)} \mid f \in \mathbb{K}[X], 1+g \in S_{>}\right\} .
$$

We have $\operatorname{Loc}_{>}(\mathbb{K}[X])=\mathcal{O}_{p=0}$. Under the local order, there is a difficulty for the successive reductions, because we may have an infinite strictly decreasing sequence of terms. For example, consider the polynomials of one variable $X, f=X$ and $g=X-X^{2}$, and we divide $f$ by $g$ by using the division algorithm, so that we successively reduce by $X-X^{2}$. This gives the reductions:

$$
\begin{aligned}
f_{1} & :=\operatorname{Red}(f, g)=X^{2} \\
f_{2} & :=\operatorname{Red}\left(f_{1}, g\right)=X^{3} \\
& \vdots \\
f_{n} & :=\operatorname{Red}\left(f_{n-1}, g\right)=X^{n+1},
\end{aligned}
$$

and so on.

Mora introduced a method to solve this problem. The following result can be found in [6].

Theorem 3.1 (Mora normal form algorithm).

Given non-zero polynomials $f, f_{1}, \ldots, f_{s} \in \mathbb{K}[X]$ and let $>$ be a local order. There is an algorithm which gives the polynomials $u, a_{1}, \ldots, a_{s}, h \in \mathbb{K}[X]$ such that

$$
u f=a_{1} f_{1}+\cdots+a_{s} f_{s}+h
$$

where $\mathrm{lt}_{>}(u)=1\left(u=1+g\right.$ is unit in $\left.\operatorname{Loc}_{>}(\mathbb{K}[X])\right)$, lt $>(f) \geq \mathrm{lt}_{>}\left(a_{i}\right) \cdot \mathrm{lt}_{>}\left(f_{i}\right)$ for all $i$ with $a_{i} \neq 0$ and $h=0$ or $\mathrm{lt}_{>}(h)$ is not divisible by any of $\mathrm{lt}_{>}\left(f_{i}\right)$. We denote $h$ by $N F(f \mid G)$ with $G=\left\{f_{1}, \ldots, f_{s}\right\}$ and we say that $h$ is the weak normal form of $f$ on division by $G$.

For $f \in \mathbb{K}[X]$, we define ecart $(f):=\operatorname{deg}(f)-\operatorname{deg}\left(\operatorname{lt}_{>}(f)\right)$. The remainder $h$ in (3.2) is produced by the following algorithm called Mora's division algorithm

$$
\begin{gathered}
h:=f ; L:=\left\{f_{1}, \ldots, f_{s}\right\} ; M:=\left\{g \in L \mid \mathrm{lt}_{>}(g) \text { divides } \mathrm{lt}_{>}(h)\right\} \\
\text { WHILE }(h \neq 0 A N D M \neq \emptyset) \mathrm{DO} \\
\text { SELECT } g \in M \text { with ecart }(g) \text { minimal } \\
\text { IF ecart }(g)>\operatorname{ecart}(h) \text { THEN } \\
L:=L \cup\{h\} \\
h:=\operatorname{Red}(h, g) \\
\text { IF } h \neq 0 T H E N \\
M:=\left\{g \in L \mid \mathrm{lt}_{>}(g) \text { divides } \mathrm{lt}_{>}(h)\right\}
\end{gathered}
$$




\section{Linear codes and binomial ideals}

Let $\mathbb{F}_{p}$ be the finite field with $p$ elements where $p$ is a prime number. A linear code $\mathcal{C}$ of length $n$ and dimension $k$ over $\mathbb{F}_{p}$ is the image of a linear, injective mapping

$$
\psi: \mathbb{F}_{p}^{k} \longrightarrow \mathbb{F}_{p}^{n}
$$

where $k \leq n$. The elements $x=\left(x_{1}, \ldots, x_{n}\right) \in \mathcal{C}$ are called the codewords. The weight of a word $x=\left(x_{1}, \ldots, x_{n}\right) \in \mathbb{F}_{p}^{n}$ is defined by $w_{t}(x):=\operatorname{card}\left\{i \mid x_{i} \neq 0,1 \leq i \leq n\right\}$. The minimum distance of the linear code $\mathcal{C}$ is $d:=\min \{d(x, y) \mid$ $x, y \in \mathcal{C}, x \neq y\}$ or $d:=\min \left\{w_{t}(x) \mid x \in \mathcal{C}, x \neq 0\right\}$ where $d(x, y):=\operatorname{card}\left(\left\{i \mid x_{i} \neq y_{i}\right\}\right)$. We define the support of an element $x \in \mathcal{C}$ by $\operatorname{supp}(x):=\left\{i \mid x_{i} \neq 0\right\}$. A linear code $\mathcal{C}$ of length $n$ and dimension $k$ is called an $[n, k]-$ code. Moreover, if the minimum distance is $d$, we say that $\mathcal{C}$ is an $[n, k, d]-$ code.

Let $\mathcal{C}$ be an $[n, k]-$ code, $e_{i}=\left(\zeta_{i 1}, \ldots, \zeta_{i k}\right)$ where $i=1, \ldots, k$ the canonical basis of $\mathbb{F}_{p}^{k}$ and

$\psi\left(e_{i}\right)=\left(g_{i 1}, \ldots, g_{i n}\right)$. The generating matrix of $\mathcal{C}$ is the matrix of dimension $k \times n$ defined by $G=\left(g_{i j}\right)$ where $g_{i j} \in \mathbb{F}_{p}$. The linear code $\mathcal{C}$ is represented as follows $\mathcal{C}=\left\{x G \mid x \in \mathbb{F}_{p}^{k}\right\}$. We will say that $G$ is in standard form if $G=\left(I_{k} \mid M\right)$ where $I_{k}$ is the $k \times k$ identity matrix.

Let $\mathcal{C}$ be an $[n, k]-$ code over $\mathbb{F}_{p}$. Define the ideal associated with $\mathcal{C}$ as $([2,8,10,16])$

$$
I_{\mathcal{C}}:=\left\langle X^{c}-X^{c^{\prime}} \mid c-c^{\prime} \in \mathcal{C}\right\rangle+\left\langle X_{i}^{p}-1 \mid 1 \leq i \leq n\right\rangle
$$

where each word $c \in \mathbb{F}_{p}^{n}$ is considered as an integral vector in the monomial $X^{c}$. Let $\mathcal{C}$ be an $[n, k]-\operatorname{code}$ over $\mathbb{F}_{p}$ and

$$
G=\left(g_{i j}\right)=\left(I_{k} \mid M\right)
$$

a generating matrix in standard form . Let $m_{i}$ be the vector of length $n$ over $\mathbb{F}_{p}$ defined by

$$
m_{i}=\left(0, \ldots, 0, p-g_{i, k+1}, \ldots, p-g_{i, n}\right)
$$

for $1 \leq i \leq k$. We have $X^{m_{i}}=X_{k+1}^{p-g_{i, k+1}} X_{k+2}^{p-g_{i, k+2}} \ldots X_{n}^{p-g_{i, n}}=\prod_{j \in \operatorname{supp}\left(m_{i}\right)} X_{j}^{p-g_{i, j}}$. In particular, if $\operatorname{supp}\left(m_{i}\right)$ is the empty set, then $X^{m_{i}}=1$.

Theorem 4.1. Let us take the lexicographic order on $\mathbb{K}[X]$ with $X_{1}>X_{2}>\cdots>X_{n}$. The code ideal $I_{\mathcal{C}}$ has the reduced Groebner basis

$$
\mathcal{G}=\left\{X_{i}-X^{m_{i}} \mid 1 \leq i \leq k\right\} \cup\left\{X_{i}^{p}-1 \mid k+1 \leq i \leq n\right\} .
$$

Proof. A proof can be found in [15].

\section{Standard bases}

In this section, we will describe the analogues of Groebner bases called standard bases for the ideals in local rings by Mora's division algorithm. Given any local order $>$ on monomials in $\mathbb{K}[X]$, there is a natural extension of $>$ to $\operatorname{Loc}_{>}(\mathbb{K}[X])$, which we will also denote by $>$. For any $h=\frac{f}{1+g} \in \operatorname{Loc}_{>}(\mathbb{K}[X])$ as in (3.1), we define $\operatorname{lm}_{>}(h):=\operatorname{lm}_{>}(f), \operatorname{lc}_{>}(h):=\operatorname{lc}_{>}(f)$ and $\operatorname{lt}_{>}(h):=\operatorname{lt}_{>}(f)$. We fix a local order $>$ on $\operatorname{Loc}_{>}(\mathbb{K}[X])$ and let $I$ be an ideal in $\operatorname{Loc}_{>}(\mathbb{K}[X])$. A standard basis of $I$ is a subset $\left\{f_{1}, \ldots, f_{r}\right\}$ of $I$ such that $l \mathrm{lt}_{>}(I)=\left\langle\mathrm{lt}_{>}\left(f_{1}\right), \ldots, \mathrm{lt}_{>}\left(f_{r}\right)\right\rangle$ where $\mathrm{lt}_{>}(I)$ is the ideal generated by the set of $\mathrm{lt}_{>}(f)$ with $f \in I$.

Proposition 5.1 (Product criterion). [11]

Let $f, g \in \mathbb{K}\left[X_{1}, \ldots, X_{n}\right]$ be polynomials such that

$\operatorname{lcm}\left(\operatorname{lm}_{>}(f), \operatorname{lm}_{>}(g)\right)=\operatorname{lm}_{>}(f) \cdot \operatorname{lm}_{>}(g)$, then

$$
N F(\operatorname{spoly}(f, g) \mid\{f, g\})=0
$$

where NF(- -$)$ is defined as in Theorem 3.1.

We will consider the ideals of the local ring $\operatorname{Loc}_{>}(\mathbb{K}[X])$ which are generated by polynomials of $\mathbb{K}[X]$. A more general result of the following theorem can be found in [11]. 
Theorem 5.1 (Buchberger criterion).

Let $I \subset \operatorname{Loc}_{>}(\mathbb{K}[X])$ be an ideal, $G=\left\{g_{1}, \ldots, g_{s}\right\}$ a set of polynomials of $I$ and $>$ a local order. Let $N F(-\mid-)$ be the weak normal form as in Theorem 3.1. Then the following are equivalent:

i) $G$ is a standard basis of $I$.

ii) G generates $I$ and $N F\left(\operatorname{spoly}\left(g_{i}, g_{j}\right) \mid G\right)=0$ for $i, j=1, \ldots, s$.

iii) G generates I and $N F\left(\operatorname{spoly}\left(g_{i}, g_{j}\right) \mid G_{i j}\right)=0$ for a suitable subset $G_{i j} \subset G$ and $i, j=1, \ldots, s$.

Let $\mathcal{C}$ be an $[n, k]-$ code, the point $(1, \ldots, 1)$ is a zero of the code ideal $I_{\mathcal{C}}$ in the affine space over $\mathbb{F}_{p}$. Rather than localizing at the maximal ideal $\left\langle X_{1}-1, \ldots, X_{n}-1\right\rangle$, we change coordinates to translate the point to the origin. Denote $I_{\mathcal{C}}^{\prime}$ the corresponding ideal, and $I:=I_{\mathcal{C}}^{\prime} \operatorname{Loc}_{>}\left(\mathbb{F}_{p}[X]\right)$ the ideal of $\operatorname{Loc}_{>}\left(\mathbb{F}_{p}[X]\right)$ generated by $I_{\mathcal{C}}^{\prime}$.

Lemma 5.1. Let $p$ a prime number, we have

$I_{\mathcal{C}}^{\prime}=\left\langle\left(X_{i}+1\right)+(p-1) \prod_{j \in \operatorname{supp}\left(m_{i}\right)}\left(X_{j}+1\right)^{p-g_{i, j}} \mid 1 \leq i \leq k\right\rangle+\left\langle\left(X_{i}+1\right)^{p}+p-1 \mid k+1 \leq i \leq n\right\rangle$ where $g_{i, j}$ is defined in (4.2) and $m_{i}$ in (4.3).

Proof. The ideal $I_{\mathcal{C}}$ defined in (4.1) has the reduced Groebner basis (4.4) by the Theorem 4.1 with respect to the lexicographic order on $\mathbb{K}[X]$. This is an ideal basis of $I_{\mathcal{C}}$ in $\mathbb{K}[X]$. The translation is made via the ring map $X_{i} \longmapsto X_{i}+1$. Since $\mathbb{K}[X] \subset \operatorname{Loc}_{>}(\mathbb{K}[X])$, then the claim for the translated ideal follows.

Now we present our main result.

Theorem 5.2. Let $\mathcal{C}$ be an $[n, k]-$ code over $\mathbb{F}_{p}$ with $p$ a prime number. Under the negative degree lexicographic order $>$ on $\mathbb{F}_{p}[X]$, the ideal $I=I_{\mathcal{C}}^{\prime} \operatorname{Loc}_{>}\left(\mathbb{F}_{p}[X]\right)$ in $\operatorname{Loc}_{>}\left(\mathbb{F}_{p}[X]\right)$ has the standard basis

$$
S=\left\{X_{i}-\sum_{\substack{0 \leq t_{l} \leq p-g_{i, j}\left(1 \leq l \leq \sigma_{i}\right) \\
\left(t_{1}, \ldots, t_{\sigma_{i}}\right) \neq(0, \ldots, 0)}} \prod_{h=1}^{\sigma_{i}}\left(\begin{array}{c}
p-g_{i, j_{h}} \\
t_{h}
\end{array}\right) X_{j_{h}}^{t_{h}} \mid 1 \leq i \leq k\right\} \cup\left\{X_{i}^{p} \mid k+1 \leq i \leq n\right\}
$$

where $\sigma_{i}:=\operatorname{card}\left(\operatorname{supp}\left(m_{i}\right)\right)$ is the number of elements in $\operatorname{supp}\left(m_{i}\right)$.

Proof. We will show that $S$ generates $I_{\mathcal{C}}^{\prime}$, then we prove that $S$ is a standard basis.

For $k+1 \leq i \leq n$

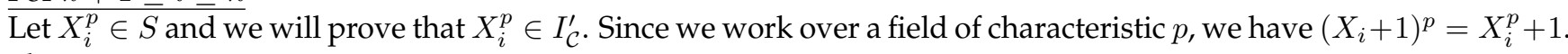
Thus

$$
X_{i}^{p}=\left(X_{i}+1\right)^{p}+p-1 \in I_{\mathcal{C}}^{\prime}
$$

For $1 \leq i \leq k$

Let $X_{i}-\sum_{\substack{0 \leq t_{i} \leq p-g_{i, j}\left(1 \leq l \leq \sigma_{i}\right) \\\left(t_{1}, \ldots, t_{\sigma_{i}}\right) \neq(0, \ldots, 0)}} \prod_{h=1}^{\sigma_{i}}\left(\begin{array}{c}p-g_{i, j_{h}} \\ t_{h}\end{array}\right) X_{j_{h}}^{t_{h}} \in S$. Suppose that

$\operatorname{supp}\left(m_{i}\right)=\left\{j_{1}, j_{2}, \ldots, j_{\sigma_{i}}\right\}$ with $j_{1}<j_{2}<\cdots<j_{\sigma_{i}}$ and denote 


$$
\begin{aligned}
& A:=X_{i}-\sum_{\substack{0 \leq t_{l} \leq p-g_{i, j_{l}}\left(1 \leq l \leq \sigma_{i}\right) \\
\left(t_{1}, \ldots, t_{\sigma_{i}} \neq(0, \ldots, 0)\right.}} \prod_{h=1}^{\sigma_{i}}\left(\begin{array}{c}
p-g_{i, j_{h}} \\
t_{h}
\end{array}\right) X_{j_{h}}^{t_{h}} \text {. Since we work over a field of characteristic } p \text {, we have } \\
& A=X_{i}+1-\left[\sum_{\substack{0 \leq t_{l} \leq p-g_{i, j_{l}}\left(1 \leq l \leq \sigma_{i}\right) \\
\left(t_{1}, \ldots, t_{\sigma_{i}}\right) \neq(0, \ldots, 0)}} \prod_{h=1}^{\sigma_{i}}\left(\begin{array}{c}
p-g_{i, j_{h}} \\
t_{h}
\end{array}\right) X_{j_{h}}^{t_{h}}+1\right] \\
& =X_{i}+1-\sum_{0 \leq t_{l} \leq p-g_{i, j_{l}}\left(1 \leq l \leq \sigma_{i}\right)} \prod_{h=1}^{\sigma_{i}}\left(\begin{array}{c}
p-g_{i, j_{h}} \\
t_{h}
\end{array}\right) X_{j_{h}}^{t_{h}} \\
& =X_{i}+1-\sum_{t_{1}=0}^{p-g_{i, j_{1}}} \ldots \sum_{t_{\sigma_{i}}=0}^{p-g_{i, j} \sigma_{i}}\left(\begin{array}{c}
p-g_{i, j_{1}} \\
t_{1}
\end{array}\right) \ldots\left(\begin{array}{c}
p-g_{i, j_{\sigma_{i}}} \\
t_{\sigma_{i}}
\end{array}\right) X_{j_{1}}^{t_{1}} \ldots X_{j_{\sigma_{i}}}^{t_{\sigma_{i}}} \\
& =X_{i}+1-\left[\sum_{t_{1}=0}^{p-g_{i, j_{1}}}\left(\begin{array}{c}
p-g_{i, j_{1}} \\
t_{1}
\end{array}\right) X_{j_{1}}^{t_{1}}\right] \ldots\left[\sum_{t_{\sigma_{i}}=0}^{p-g_{i, j \sigma_{i}}}\left(\begin{array}{c}
p-g_{i, j_{\sigma_{i}}} \\
t_{\sigma_{i}}
\end{array}\right) X_{j_{\sigma_{i}}}^{t_{\sigma_{i}}}\right] \\
& =X_{i}+1-\left[\left(X_{j_{1}}+1\right)^{p-g_{i, j_{1}}}\right] \ldots\left[\left(X_{j_{\sigma_{i}}}+1\right)^{p-g_{i, j_{\sigma_{i}}}}\right] \\
& =X_{i}+1-\prod_{j \in \operatorname{supp}\left(m_{i}\right)}\left(X_{j}+1\right)^{p-g_{i, j}} \\
& =X_{i}+1+p\left[\prod_{j \in \operatorname{supp}\left(m_{i}\right)}\left(X_{j}+1\right)^{p-g_{i, j}}\right]-\prod_{j \in \operatorname{supp}\left(m_{i}\right)}\left(X_{j}+1\right)^{p-g_{i, j}} \\
& =X_{i}+1+(p-1)\left[\prod_{j \in \operatorname{supp}\left(m_{i}\right)}\left(X_{j}+1\right)^{p-g_{i, j}}\right] \in I_{\mathcal{C}}^{\prime}
\end{aligned}
$$

By Lemma 5.1, $S$ is a generating set for $I_{\mathcal{C}}^{\prime}$.

Let us now show that $S$ is a standard basis over $\mathbb{F}_{p}$.

* Let the pair $(i, j)$ such that $k+1 \leq i<j \leq n$.

We have $\operatorname{spoly}\left(X_{i}^{p}, X_{j}^{p}\right)=\frac{X_{i}^{p} X_{j}^{p}}{X_{i}^{p}} X_{i}^{p}-\frac{X_{i}^{p} X_{j}^{p}}{X_{j}^{p}} X_{j}^{p}=X_{i}^{p} X_{j}^{p}-X_{i}^{p} X_{j}^{p}=0$, and $N F(0 \mid S)=0$.

* Let the pair $(i, j)$ such that $1 \leq i \leq k$ and $k+1 \leq j \leq n$.

$$
\begin{aligned}
& \text { Denote } \quad f_{i}:=X_{i}-\sum_{\substack{0 \leq t_{l} \leq p-g_{i, j_{l}}\left(1 \leq l \leq \sigma_{i}\right) \\
\left(t_{1}, \ldots, t_{\sigma_{i}}\right) \neq(0, \ldots, 0)}} \prod_{h=1}^{\sigma_{i}}\left(\begin{array}{c}
p-g_{i, j_{h}} \\
t_{h}
\end{array}\right) X_{j_{h}}^{t_{h}} \text {. We have } \\
& \operatorname{spoly}\left(f_{i}, X_{j}^{p}\right)=\frac{X_{i} X_{j}^{p}}{X_{i}}\left[X_{i}-\sum_{\substack{0 \leq t_{l} \leq p-g_{i, j_{l}}\left(1 \leq l \leq \sigma_{i}\right) \\
\left(t_{1}, \ldots, t_{\sigma_{i}}\right) \neq(0, \ldots, 0)}} \prod_{h=1}^{\sigma_{i}}\left(\begin{array}{c}
p-g_{i, j_{h}} \\
t_{h}
\end{array}\right) X_{j_{h}}^{t_{h}}\right]-\frac{X_{i} X_{j}^{p}}{X_{j}^{p}} X_{j}^{p} \\
& =X_{i} X_{j}^{p}-X_{j}^{p}\left[\sum_{\substack{0 \leq t_{l} \leq p-g_{i, j_{l}}\left(1 \leq l \leq \sigma_{i}\right) \\
\left(t_{1}, \ldots, t_{\sigma_{i}} \neq(0, \ldots, 0)\right.}} \prod_{h=1}^{\sigma_{i}}\left(\begin{array}{c}
p-g_{i, j_{h}} \\
t_{h}
\end{array}\right) X_{j_{h}}^{t_{h}}\right]-X_{i} X_{j}^{p} \\
& =-X_{j}^{p}\left[\sum_{\substack{0 \leq t_{l} \leq p-g_{i, j_{l}}\left(1 \leq l \leq \sigma_{i}\right) \\
\left(t_{1}, \ldots, t_{\sigma_{i}}\right) \neq(0, \ldots, 0)}} \prod_{h=1}^{\sigma_{i}}\left(\begin{array}{c}
p-g_{i, j_{h}} \\
t_{h}
\end{array}\right) X_{j_{h}}^{t_{h}}\right]
\end{aligned}
$$

In the last expression, all these monomials are multiple of $X_{j}^{p} \in S$. Therefore the remainder of the division of $\operatorname{spoly}\left(f_{i}, X_{j}^{p}\right)$ by $\left\{X_{j}^{p}\right\}$ is zero, i.e $N F\left(\operatorname{spoly}\left(f_{i}, X_{j}^{p}\right) \mid\left\{X_{j}^{p}\right\}\right)=0$. 
* Finally, let $1 \leq i<j \leq k$.

$$
\text { Let } g_{i}:=X_{i}-\sum_{\substack{0 \leq t_{l} \leq p-g_{i, r_{l}}\left(1 \leq l \leq \sigma_{i}\right) \\
\left(t_{1}, \ldots, t_{\sigma_{i}}\right) \neq(0, \ldots, 0)}} \prod_{h=1}^{\sigma_{i}}\left(\begin{array}{c}
p-g_{i, r_{h}} \\
t_{h}
\end{array}\right) X_{r_{h}}^{t_{h}}
$$

We have $\operatorname{lt}_{>}\left(g_{i}\right)=X_{i}$ and $\mathrm{lt}_{>}\left(g_{j}\right)=X_{j}$, then $\operatorname{lcm}\left(\operatorname{lm}_{>}\left(g_{i}\right), \operatorname{lm}_{>}\left(g_{j}\right)\right)=\operatorname{lm}_{>}\left(g_{i}\right) \cdot \operatorname{lm}_{>}\left(g_{j}\right)$. According to the Product Criterion in Proposition 5.1, we obtain

$N F\left(\operatorname{spoly}\left(g_{i}, g_{j}\right) \mid\left\{g_{i}, g_{j}\right\}\right)=0$. And by the Buchberger's criterion in Theorem 5.1, the assertion follows.

Example 5.1. Consider the generator matrix $G=\left(g_{i j}\right)$ defined by

$$
G=\left(\begin{array}{llllll}
1 & 0 & 0 & 1 & 0 & 1 \\
0 & 1 & 0 & 2 & 1 & 0 \\
0 & 0 & 1 & 2 & 2 & 1
\end{array}\right)
$$

Under the negative degree lexicographic order $>$ on $\mathbb{F}_{3}[X]$, the ideal $I=I_{\mathcal{C}}^{\prime} \operatorname{Loc}_{>}\left(\mathbb{F}_{3}[X]\right)$ where $I_{\mathcal{C}}^{\prime}$ is defined in Lemma 5.1 with $p=3, n=6$ and $k=3$ has the standard basis

$$
S=\left\{g_{1}, g_{2}, g_{3}, X_{4}^{3}, X_{5}^{3}, X_{6}^{3}\right\}
$$

where

$$
\begin{aligned}
& g_{1}=X_{1}+X_{4}+X_{6}+2 X_{4}^{2}+2 X_{4} X_{6}+2 X_{6}^{2}+X_{4}^{2} X_{6}+X_{4} X_{6}^{2}+2 X_{4}^{2} X_{6}^{2}, \\
& g_{2}=X_{2}+2 X_{4}+X_{5}+X_{4} X_{5}+2 X_{5}^{2}+2 X_{4} X_{5}^{2}, \\
& g_{3}=X_{3}+2 X_{4}+2 X_{5}+X_{6}+2 X_{4} X_{5}+X_{4} X_{6}+X_{5} X_{6}+2 X_{6}^{2}+X_{4} X_{5} X_{6}+2 X_{4} X_{6}^{2}+2 X_{5} X_{6}^{2}+2 X_{4} X_{5} X_{6}^{2} .
\end{aligned}
$$

An immediate consequence is the result in [9] ( for $p=2$ )

Theorem 5.3. In view of the negative degree lexicographic order $>$ on $\mathbb{F}_{2}[X]$, the ideal $I=I_{\mathcal{C}}^{\prime}$ Loc $_{>}\left(\mathbb{F}_{2}[X]\right)$ in Loc $>\left(\mathbb{F}_{2}[X]\right)$ where

$I_{\mathcal{C}}^{\prime}=\left\langle\left(X_{i}+1\right)+\prod_{j \in \operatorname{supp}\left(m_{i}\right)}\left(X_{j}+1\right) \mid 1 \leq i \leq k\right\rangle+\left\langle\left(X_{i}+1\right)^{2}+1 \mid k+1 \leq i \leq n\right\rangle$

has the standard basis

$$
S=\left\{X_{i}-\sum_{\substack{J \subseteq \operatorname{supp}_{J \neq \emptyset}\left(m_{i}\right) \\ J \neq}} X_{J} 1 \leq i \leq k\right\} \cup\left\{X_{i}^{2} \mid k+1 \leq i \leq n\right\}
$$

\section{References}

[1] Adams, W. and Loustaunau, P., An Introduction to Groebner Bases, American Mathematical Society, Vol.3, 1994.

[2] Borges-quintana, M., Borges-Trenard, M., Fitzpatrick P. and Martinez-Moro E., Groebner bases and combinatorics for binary codes, Applicable Algebra in Engineering Communication and Computing - AAECC 19(2008), 393-411.

[3] Buchberger, B., An Algorithm for Finding the Basis Elements of the Residue Class Ring Modulo a Zero Dimensional Polynomial Ideal, PhD thesis, University of Innsbruck, 1965.

[4] Cooper, A., Towards a new method of decoding Algebraic codes using Groebner bases, Transactions 10th Army Conf. Appl. Math. Comp. 93(1992), 293-297. 
[5] Cox, D., Little J. and O'Shea D., Ideals, Varieties, and Algorithms, Springer, 1996.

[6] Cox, D., Little J. and O'Shea D., Using Algebraic Geometry, Springer, 1998.

[7] Drton, M., Sturmfels B., Sullivan S., Lectures on Algebraic Statistics, Birkhäuser, Basel, 2009.

[8] Dück, N. and Zimmermann, K.H., Gröbner bases for perfect binary linear codes, International Journal of Pure and Applied Mathematics 91(2014), no.2, 155-167.

[9] Dück, N. and Zimmermann, K.H., Standard Bases for binary Linear Codes, International Journal of Pure and Applied Mathematics 80(2012), no.3, 315-329.

[10] Dück, N. and Zimmermann, K.H., Universal Groebner bases for Binary Linear Code, International Journal of Pure and Applied Mathematics 86(2013), no.2, 345-358.

[11] Greuel, G.M. and Pfister, G., A Singular Introduction to Commutative Algebra, Springer-Verlag, Berlin, 2002.

[12] Hironaka, H., Resolution of singularities of an algebraic variety over a field of characteristic zero, Ann.Math. 79(1964), 109-326.

[13] Mora, T., Pfister, G. and Traverso, C., An introduction to the tangent cone algorithm, Advances in Computing Research 6(1992), 199-270.

[14] Sala, M., Mora, T., Perret, L., Sakata, S., and Traverso, C., Groebner Bases, Coding, and Cryptography, Springer, Berlin 2009.

[15] Saleemi, M. and Zimmermann, K.H., Groebner Bases for Linear Codes, International Journal of Pure and Applied Mathematics 62(2010), no.4, 481-491.

[16] Saleemi, M. and Zimmermann, K.H., Linear Codes as Binomial Ideals, International Journal of Pure and Applied Mathematics 61(2010), no.2, 147-156.

\section{Affiliations}

JEAN JACQUES FERDINAND RANDRIAMIARAMPANAHY

AdDRess: University of Antananarivo, Dept. of Mathematics and Computer Science, 101, AntananarivoMadagascar.

E-MAIL: randriamiferdinand@gmail.com

ORCID ID: 0000-0001-5187-4208

HARINAIVO ANDRIATAHINY

AdDress: University of Antananarivo, Dept. of Mathematics and Computer Science, 101, AntananarivoMadagascar.

E-MAIL: hariandriatahiny@gmail.com

ORCID ID: 0000-0003-4847-3854

TOUSSAINT JOSEPH RABEHERIMANANA

AdDress: University of Antananarivo, Dept. of Mathematics and Computer Science, 101, AntananarivoMadagascar.

E-MAIL: rabeherimanana.toussaint@yahoo.fr

ORCID ID: 0000-0003-0453-6403 